\title{
Oxygen therapy and inpatient mortality in COPD exacerbation
}

\author{
Carlos Echevarria (1) , ${ }^{1,2}$ John Steer, ${ }_{1}^{2,3}$ James Wason, ${ }^{4}$ Stephen Bourke ${ }^{2,3}$
}

\begin{abstract}
Handling editor Richard John Parris

${ }^{1}$ Respiratory Department, Royal Victoria Infirmary, Newcastle upon Tyne, UK

${ }^{2}$ ICM, Newcastle University, Newcastle upon Tyne, UK

${ }^{3}$ Respiratory Department, North Tyneside General Hospital, North Shields, UK

${ }^{4}$ Population Health Sciences Institute, Newcastle University, Newcastle upon Tyne, UK
\end{abstract}

\section{Correspondence to}

Professor Stephen Bourke, North Tyneside General Hospital, North Shields NE29 8NH, UK; Stephen.Bourke@nhct.nhs.uk

Received 3 November 2019 Revised 15 October 2020 Accepted 19 October 2020 Published Online First 26 November 2020

\section{SLinked}

- http://dx.doi.org/10.1136/ emermed-2020-210749

Check for updates

(C) Author(s) (or their employer(s)) 2021. No commercial re-use. See rights and permissions. Published by BMJ.

To cite: Echevarria C, Steer J, Wason J, et al. Emerg Med J 2021:38:170-177.

\section{ABSTRACT}

Background In hospitalised patients with exacerbation of Chronic Obstructive Pulmonary Disease, European and British guidelines endorse oxygen target saturations of $88 \%-92 \%$, with adjustment to $94 \%-98 \%$ if carbon dioxide levels are normal. We assessed the impact of admission oxygen saturation level and baseline carbon dioxide on inpatient mortality.

Methods Patients were identified from the prospective Dyspnoea, Eosinopenia, Consolidation, Acidaemia and Atrial Fibrillation (DECAF) derivation study (December 2008-June 2010) and the mixed methods DECAF validation study (January 2012 to May 2014). In six UK hospitals, of 2645 patients with COPD exacerbation, 1027 patients were in receipt of supplemental oxygen at admission. All had a clinical history of COPD and obstructive spirometry. These patients were subdivided into the following groups: admission oxygen saturations of $87 \%$ or less, $88 \%-92 \%, 93 \%-96 \%$ or $97 \%-100 \%$. Inpatient mortality was calculated for each group and expressed as ORs. The DECAF score and National Early Warning Score 2 (excluding oxygen saturation) were used in binary logistic regression to adjust for baseline risk.

Results In patients with COPD receiving supplemental oxygen, oxygen saturations above $92 \%$ were associated with higher mortality and an adverse dose-response. Compared with the $88 \%-92 \%$ group, the adjusted risk of death (OR) in the $93 \%-96 \%$ and $97 \%-100 \%$ groups was $1.98(95 \% \mathrm{Cl} 1.09$ to $3.60, \mathrm{p}=0.025)$ and 2.97 $(95 \% \mathrm{Cl} 1.58$ to $5.58, \mathrm{p}=0.001)$. In the subgroup with normocapnia, the mortality signal remained significant in both the $93 \%-96 \%$ and $97 \%-100 \%$ groups.

Conclusions Inpatient mortality was lowest in those with oxygen saturations of $88 \%-92 \%$. Even modest elevations in oxygen saturations above this range $(93 \%-96 \%)$ were associated with an increased risk of death. A similar mortality trend was seen in both patients with hypercapnia and normocapnia. This shows that the practice of setting different target saturations based on carbon dioxide levels is not justified. Treating all patients with COPD with target saturations of $88 \%-92 \%$ will simplify prescribing and should improve outcome. Trial registration number UKCRN ID 14214.

\section{INTRODUCTION}

In patients admitted to hospital with an exacerbation of Chronic Obstructive Pulmonary Disease (ECOPD), appropriate oxygen therapy can save lives, but excess oxygenation is associated with higher rates of ventilation ${ }^{12}$ and death. ${ }^{3}$ Supranormal oxygen levels can cause significant harm, but the degree of harm in patients with COPD at
Key messages

What is already known on this topic

- The European and British Thoracic Societies guidelines endorse target oxygen saturations of $88 \%-92 \%$ in patients admitted with COPD exacerbation, which can be adjusted to $94 \%$ $98 \%$ following confirmation of normocapnia.

- Conversely, the National Early Warning Score 2 guidelines recommend target saturations of $94 \%-98 \%$, with adjustment to $88 \%-92 \%$ following confirmation of hypercapnia and under the guidance of a clinician.

- At the time of our study, the medical literature shows that excess oxygen is harmful in patients with COPD, in part due to hypercapnia.

\section{What this study adds}

- In an observational study of 2645 consecutive patients admitted with COPD exacerbation to 1 of 6 UK hospitals, 1027 had oxygen therapy at admission. In those treated with oxygen, saturations above $88 \%-92 \%$ had an incrementally higher risk-adjusted in-hospital mortality, both overall and in the subgroup with normocapnia.

- Contrary to current recommendations and clinical dogma, patients with COPD exacerbations and baseline normocapnia, who receive supplementary oxygen therapy, are at considerable risk of death with elevated oxygen saturations.

- Adopting target saturations $88 \%-92 \%$ for all hospitalised patients with COPD would simplify clinical pathways and should improve outcomes.

oxygen levels that would be regarded as normal in healthy individuals is unclear. Oxygen guidelines conflict in their recommended target range for oxygen saturations and the need to adjust this range in the presence of hypercapnia. ${ }^{4-12}$

The British Thoracic Society (BTS) recommends initial target oxygen saturations of $88 \%-92 \%$, which can be increased to $94 \%-98 \%$ once normocapnia has been confirmed. ${ }^{10}$ This guidance was endorsed by the European Thoracic Society (ERS), ${ }^{12}$ although subsequent joint ERS and American Thoracic Society (ATS) guidelines lack a target range. ${ }^{13} \mathrm{In}$ contrast, the Global Initiative for Obstructive Lung Disease $2019^{14}$ and Thoracic Society of Australia and New Zealand ${ }^{15}$ recommend target saturations 88\%-92\% with no caveat for hypercapnia. The 
ERS/ATS non-invasive ventilation (NIV) guidelines include a target of 88\%-92\% for those with hypercapnia. ${ }^{16}$

The National Early Warning Score (NEWS) is used to monitor patients for clinical deterioration using physiological indices that predict short-term death. The updated NEWS2 includes an additional oxygen saturation scale for those with hypercapnic respiratory failure $\left(\mathrm{PaCO}_{2}>45 \mathrm{~mm} \mathrm{Hg}\right.$ or $\left.6 \mathrm{kPa}\right){ }^{11}$ This aims to mitigate the harms of excess oxygen by promoting target saturations of $88 \%-92 \%$. However, the new scale requires proof of hypercapnia and clinician approval which may paradoxically result in more patients with COPD initially receiving the default 94\%-98\% range.

Oxygen-induced hypercapnia is a dangerous and complex phenomenon caused primarily by ventilation-perfusion mismatching and changes in minute ventilation. ${ }^{16}$ It is not restricted to patients who are already hypercapnic, ${ }^{17}$ and may lead to respiratory acidaemia and death. Excess oxygen additionally causes atelectasis, inflammation, acute lung injury and cardiac and cerebral vasoconstriction. ${ }^{9}{ }^{18} 19$

An randomised controlled trial (RCT) performed in Australia in patients with presumed ECOPD showed high-flow oxygen substantially increased mortality compared with target saturations of $88 \%-92 \% .{ }^{20}$ Mortality was lower with target therapy even in patients without spirometry confirmed COPD, although non-significantly. A 2018 meta-analysis showed liberal oxygen was associated with a dose-dependent increase in mortality across a range of medical conditions. ${ }^{21}$

Regrettably, injudicious oxygen use remains extremely common. A European audit in 2012 showed that one in four patients with ECOPD received high-flow oxygen prior to admission. ${ }^{22}$ UK-based observational studies from $2012^{23}$ and $2017^{1}$ showed that overoxygenation occurred in three-quarters and one-half of patients, respectively. The latter study focused on patients receiving acute NIV; ECOPD was the most common indication and oxygen toxicity contributed to approximately a quarter of all episodes of acute hypercapnic respiratory failure.

Previous studies show that healthcare workers do not understand the indications and harms of supplemental oxygen, and have entrenched views of its presumed benefit ${ }^{24}$; the conflicting advice between guidelines, with differing caveats for the 'acutely ill' and normocapnic, may add to the confusion.

In this study, we examined in-hospital mortality in patients with ECOPD across admission oxygen saturation groups and by receipt of supplemental oxygen. Furthermore, we further assess mortality, $\mathrm{pH}$ and $\mathrm{PaCO}_{2}$ in patients with baseline normocapnia to see if the current practice of targeting such patients with higher oxygen saturations is justified. For simplicity, we define normocapnia as any patient who is not hypercapnic.

\section{METHODS}

The main aim of this study was to examine in-hospital mortality in ECOPD, first across admission oxygen saturation groups of (1) $87 \%$ or less, (2) $88 \%-92 \%$, (3) $93 \%-96 \%$ or (4) $97 \%-100 \%$ in (a) those receiving oxygen and (b) those not receiving oxygen at admission, and second in the subgroup of patients with hypercapnia and normocapnia. Inpatient mortality was the primary outcome, and 1-year mortality was the secondary outcome.

The Dyspnoea, Eosinopenia, Consolidation, Acidaemia and Atrial Fibrillation (DECAF) score predicts risk of in-hospital death in ECOPD, outperforming alternative tools. ${ }^{25}{ }^{26}$ The DECAF derivation and validation cohorts, comprising 2645 consecutive admissions of unique patients with ECOPD to one of six UK hospitals, were used in this study. ${ }^{25}$ The DECAF derivation and external validation cohorts were prospective, though the internal validation study included a mix of prospective and retrospective patients (collection of DECAF was part of usual care). All patients had obstructive spirometry performed prior to admission as part of usual care, an age of 35 years or older and a smoking history of 10 or more cigarette pack years. Exclusion criteria were any illness, other than COPD, that was likely to limit survival to less than 1 year. Approximately $3 \%$ of patients were excluded for survival limitations, principally malignancy. A prespecified requirement of the DECAF studies was robust collection of admission oxygen saturations, other candidate predictors, and inpatient death (the primary outcome). Therefore, these cohorts are well placed to explore the hypothesis that admission oxygen saturations are associated with inpatient death and adjust for baseline risk.

The DECAF and NEWS2 indices (respiratory rate, oxygen saturations, systolic blood pressure, pulse rate, level of consciousness and confusion and temperature) were collected at admission. The first admission oxygen saturation measurement was used, whether taken in accident and emergency or the medical admissions unit, which typically occurred at triage. Arterial blood analysis (ABG) was performed as part of usual care, usually within the first few hours of attendance to accident and emergency or the medical admissions unit. Patients were followed up to 1 year or death. Inpatient deaths were reported by the clinical team, and deaths were triangulated with the hospital Patient Administration System.

Missing data were handled using multiple imputation as described previously. ${ }^{2728}$ For missing data related to oxygen saturations group, data were imputed based on ABG results. Patients were divided into the following four admission oxygen saturations groups: (1) $87 \%$ or less, (2) $88 \%-92 \%$, (3) $93 \%-96 \%$ or (4) 97\%-100\%. In-hospital mortality was compared across these four groups in those with and without supplemental oxygen.

ORs and 95\% CIs were calculated using logistic regression for univariate and multivariate analyses. For multivariate analysis, baseline inpatient risk of death was adjusted using patient's DECAF scores and their admission physiological indices, as per NEWS2 but excluding oxygen saturation (NEWS2-sats). The DECAF score was the best predictor of inpatient death in this cohort, ${ }^{25} 26$ and NEWS2 adds additional prognostic information, which justifies their selection for the adjusted model. As requested by a reviewer, we also applied a method that allows for estimating the relative risk instead of the OR for the multivariate model. We initially applied Poisson regression, but it was apparent from the model fit that the data were underdispersed. We therefore applied Conway-Maxwell-Poisson regression which better allows for underdispersion. ${ }^{29}$

The e value was calculated for adjusted risks and provides evidence for causality in observational studies. ${ }^{30}$ It is a quantitative measure of the Bradford Hill criterion that states stronger associations make causality more likely. It represents the required minimum strength of an association between a hypothetical unmeasured covariate and both the treatment (in this case oxygen) and outcome (inpatient death) to fully explain the observed association. A large e value implies it is less likely that the results are due to the confounding effects of an unmeasured variable.

Patients with target saturations of $88 \%-92 \%$ are at risk of dropping below this range and so mortality in the oxygen saturation range $85 \%-87 \%$ was separately assessed. In those who survived to discharge, 1-year mortality was presented by oxygen saturation group. Analyses were performed using IBM SPSS V.22. 


\section{Patient and public involvement}

This programme of research has included interviews with patients hospitalised with ECOPD, and liaison with an expert patient.

\section{RESULTS}

\section{Population and missing data}

There were 2645 patients from the DECAF cohorts, the characteristics of whom are described elsewhere. ${ }^{25}{ }^{26}$ One thousand twenty-seven (39\%) patients were in receipt of supplemental oxygen at admission. The mean age (SD) was 73.3 (10.2), 56\% were women, mean (SD) preadmission FEV1 was 41.7 (17.4) per cent predicted, $57 \%$ could not leave home without help, and $31 \%$ had consolidation on admission chest X-ray. Twentynine per cent had acidaemia on admission ABG and 54\% had a Charlson index of 2 or more. Inpatient mortality was $12.8 \%$, while the inpatient mortality in the 1618 patients that did not receive oxygen was $6.0 \%$

Rates of missing data were low, with rates of $5.6 \%$ for NEWS2 (temperature $4.0 \%$, respiratory rate $2.7 \%$, alert, voice, pain, unresponsive and confusion $2.5 \%$, systolic blood pressure $2.4 \%$, pulse $2.3 \%)$ and $0 \%$ for the DECAF score $(\mathrm{pH}$ was regarded as non-scoring, ie, $\geq 7.30$, in those deemed too well for ABG sampling). In all patients, $10.1 \%$ had missing $A B G$ data, but only $3.6 \%$ of those receiving supplemental oxygen had missing ABG data. Admission oxygen saturations from pulse oximetry were unavailable for $2.9 \%$. For the primary outcome, inpatient mortality, there were no missing data, and no patients were lost to follow-up.

\section{In-hospital mortality}

In-hospital mortality in patients receiving supplemental oxygen

There were 1027 patients receiving supplemental oxygen at the time admission oxygen saturations were recorded, with $14 \%$ in the $87 \%$ or less group, $28 \%$ in the $88 \%-92 \%$ group, $37 \%$ in the $93 \%-96 \%$ group and $22 \%$ in the $97 \%-100 \%$ group. Table 1 shows DECAF and NEWS2-sats indices across oxygen saturation groups. The $97 \%-100 \%$ group had a better mortality risk profile with respect to systolic blood pressure, fever, eosinopenia and consolidation. However, $\mathrm{pH}$ was lower, and both
$\mathrm{PaO}_{2}$ and $\mathrm{PaCO}_{2}$ and their SD higher in the $97 \%-100 \%$ group which may be accounted for by excess oxygen. The $87 \%$ or less group showed high variability in mean $\mathrm{PaO}_{2}$ and $\mathrm{PaCO}_{2}$ which is perhaps due to some patients being given high rates of oxygen following identification of low oxygen saturations prior to $A B G$ sampling. Further baseline data are shown in online supplemental table 1.

The lowest mortality was in the 88\%-92\%group: $88 \%-92 \%=8.7 \% ; \quad 97 \%-100 \%=17.1 \% ; \quad 93 \%-96 \%=11.7 \%$; and $87 \%$ or less $=17.1 \%$ (figure 1). Table 2 shows the risk of death across groups compared with the 88\%-92\% reference group, by OR and following adjustment for baseline mortality risk. After adjusting for baseline risk, mortality was further increased in both the $97 \%-100 \%$ group (unadjusted $\mathrm{OR}=2.18$; adjusted $\mathrm{OR}=2.97$ ) and $93 \%-96 \%$ group (unadjusted $\mathrm{OR}=1.40$; adjusted $\mathrm{OR}=1.98$ ), but less prominent in the $87 \%$ or less group (unadjusted $\mathrm{OR}=2.17$; adjusted $\mathrm{OR}=1.36$ ).

The e value for the adjusted OR for the 97\%-100\% group was 5.39 (95\% CI 2.54 to 10.6 ), and for the $93 \%-96 \%$ was 3.37 (95\% CI 1.40 to 6.66 ). Higher mortality was therefore not explained by baseline risk, supporting the hypothesis that overuse of oxygen contributed to mortality. Furthermore, an incremental, higher risk of death was seen at higher oxygen saturation levels.

In-hospital mortality in patients receiving supplemental oxygen with oxygen saturations of $85 \%-87 \%$

Patients with oxygen saturations of $85 \%-87 \%$ had an inpatient mortality of $10.4 \%$, falling between the mortality in the $88 \%-92 \%$ and $93 \%-96 \%$ groups. This may provide reassurance that dropping marginally below the $88 \%-92 \%$ threshold is not particularly unsafe, but this group is small $(\mathrm{n}=58)$, and so these results should be interpreted with caution.

In-hospital mortality in patients with hypercapnia and normocapnia receiving supplemental oxygen

Of 1027 patients receiving supplemental oxygen, 476 were normocapnic. Patients with normocapnia with admission saturations of $97 \%-100 \%$ and $93 \%-96 \%$ had significantly higher

Table 1 Baseline characteristics between admission oxygen saturation groups in those receiving supplemental oxygen

\begin{tabular}{|c|c|c|c|c|}
\hline & \multicolumn{4}{|c|}{ Admission oxygen saturation group } \\
\hline & $87 \%$ or less $n=147$ & $88 \%-92 \% n=282$ & $93 \%-96 \% n=375$ & $97 \%-100 \% n=223$ \\
\hline \multicolumn{5}{|l|}{ DECAF indices } \\
\hline eMRCD Score, (IQR) & $5 a(4-5 a)$ & $5 a(4-5 b)$ & $5 a(4-5 a)$ & $5 a(4-5 b)$ \\
\hline Eosinopenia less than $0.05, \%$ & 60.7 & 60.3 & 51.1 & 44.1 \\
\hline CXR consolidation, $\%$ & 43.0 & 33.0 & 29.0 & 25.5 \\
\hline $\mathrm{pH}$ less than $7.35 / 7.3, \%$ & $53.8 / 34.9$ & $18.9 / 9.7$ & $26.1 / 12.3$ & $31.5 / 20.1$ \\
\hline$A F, \%$ & 18.8 & 12.5 & 16.1 & 16.1 \\
\hline \multicolumn{5}{|l|}{ NEWS2-sats indices } \\
\hline Respiratory rate, (SD) & $27.1(7.7)$ & $25.0(6.4)$ & $25.2(6.5)$ & $26.3(6.9)$ \\
\hline BP, systolic, mm Hg (SD) & $136(31)$ & $135(25)$ & $139(28)$ & $141(26)$ \\
\hline Pulse rate, (SD) & $108(22)$ & $104(19)$ & $104(21)$ & $106(21)$ \\
\hline AVPU and/or confusion, $\%$ & 24.0 & 13.6 & 10.7 & 16.3 \\
\hline Temp. $38^{\circ} \mathrm{C} / 100.4 \mathrm{~F}$ or more, $\%$ & 18.3 & 13.7 & 11.9 & 6.6 \\
\hline $\mathrm{pO}_{2}, \mathrm{kPa}$ (SD) & $8.9(5.7)$ & $8.7(3.0)$ & $10.2(4.6)$ & $13.9(9.4)$ \\
\hline $\mathrm{pCO}_{2^{\prime}} \mathrm{kPa}$ (SD) & $8.4(3.5)$ & $6.6(2.4)$ & $6.6(2.1)$ & $7.2(3.2)$ \\
\hline
\end{tabular}

$A F$, atrial fibrillation; AVPU, alert, voice, pain, unresponsive; BP, blood pressure; CXR, chest radiograph; DECAF, dyspnoea, eosinopenia, chest $X$-ray consolidation, acidaemia, atrial fibrillation; eMRCD, extended Modified Research Council Dyspnoea score; kPa, kilopascals; NEWS2, National Early Warning Score; pC02, partial pressure of carbon dioxide; p02, partial pressure of oxygen; Temp., temperature. 


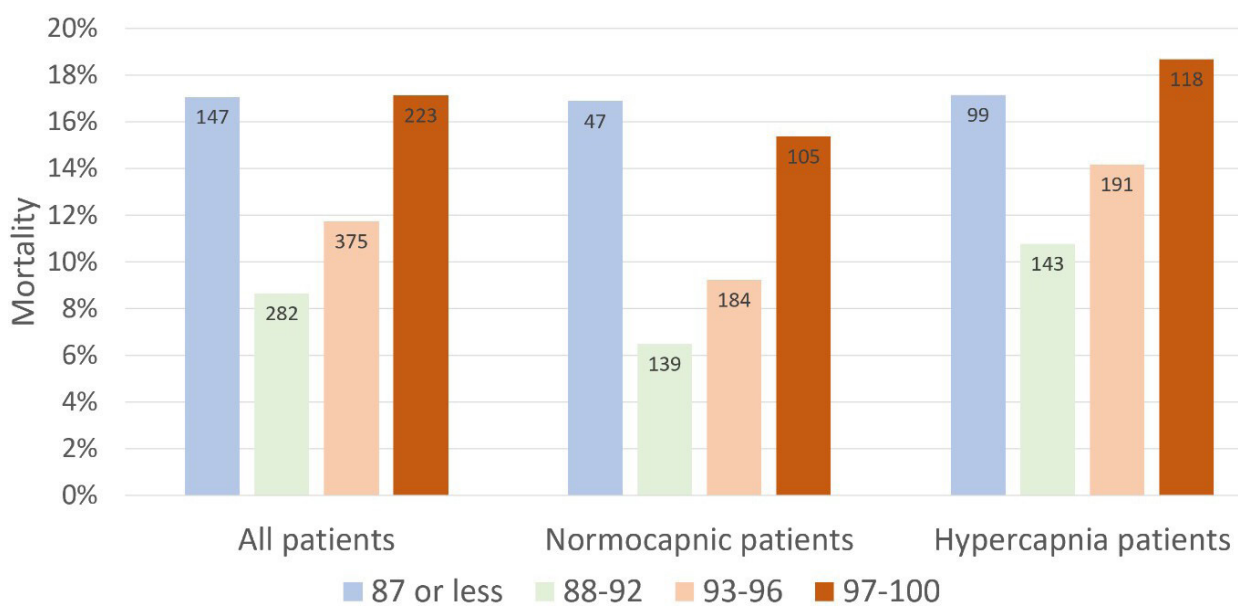

Figure 1 Inpatient mortality risk by admission oxygen saturation group in patients receiving supplemental oxygen. Patients treated with supplemental oxygen grouped by their admission oxygen saturation score, as a total $\left(\mathrm{O}_{2}\right.$ all $)$ and subdivided by $\mathrm{PaCO}_{2}\left(\mathrm{PaCO}_{2} 45 \mathrm{~mm} \mathrm{Hg} / 6.0 \mathrm{kPa}\right.$ or less and $\mathrm{PaCO}_{2}>45 \mathrm{~mm} \mathrm{Hg} / 6.0 \mathrm{kPa}$ ). The per cent inpatient mortality is shown on the y axis, and the subgroup total of patients is shown at the top of each column. Note that the numbers are rounded to the nearest integer within an imputation analysis, and hence the discrepancy between the subtotal and overall total (see appendix multiple imputation).

mortality than the 88\%-92\%group (adjusted $\mathrm{OR}=4.32$ and 2.31, respectively; figure 1 and table 3).

For patients with hypercapnia in the 97\%-100\%group, the adjusted OR was 2.28 with a $\mathrm{p}$ value of 0.052 . For patients with hypercapnia in the $93 \%-96 \%$ group, the mortality risk $(\mathrm{OR}=1.82)$ was not statistically significant.

Secondary analysis of in-hospital mortality using relative risk in patients treated with supplemental oxygen

The results for the secondary analysis showed an adjusted Risk Ratio (RR) of $2.00(95 \%$ CI 1.38 to $2.90, \mathrm{p}<0.001)$ for the 97\%-100\% group, and 1.36 (95\% CI 0.92 to $2.02, \mathrm{p}=0.127$ ). Unlike the primary analysis, only the $97 \%-100 \%$ group reached statistical significance.

In those with normocapnia, the mortality association was consistent with the primary analysis, with a significant increase in risk for both the $97 \%-100 \%$ and $93 \%-96 \%$ groups $(\mathrm{RR}=3.27$, $95 \%$ CI 1.66 to $6.45, \mathrm{p}=0.001$; and RR $2.28,95 \%$ CI 1.15 to $4.51, \mathrm{p}=0.018)$.

For those with hypercapnia, the mortality increase in the 97\%-100\% was of borderline consistency, though the 93\%-96\% did not show an association with mortality $(\mathrm{RR}=1.60,95 \% \mathrm{CI}$ 1.07 to $2.40, \mathrm{p}=0.025$; RR $1.08,95 \% \mathrm{CI} 0.70$ to $1.69, \mathrm{p}=0.721$ ).
Admission saturations and mortality in patients not treated with supplemental oxygen

In 1618 patients not treated with supplemental oxygen, the higher mortality in the $88 \%-92 \%$ group was due to a better baseline risk (tables 1 and 4, and online table 4). Mortality fell incrementally from the $88 \%-92 \%$ to the $97 \%-100 \%$ group (figure 2, right panel) in-line with a fall in $\mathrm{pCO}_{2}$. Conversely, in those treated with oxygen, the U-shaped mortality pattern was matched by a U-shaped distribution of mean $\mathrm{pCO}_{2}$ and proportion of patients with a $\mathrm{pH}$ of less than 7.35 (figure 2, left panel).

\section{One-year mortality}

Mortality at 1-year post discharge in those treated with supplemental oxygen

In patients who received supplemental oxygen on admission and survived to discharge, the risk of death at 1 year was $28 \%$. The 12-month mortality rates, excluding inpatient deaths, were: $87 \%$ or less $=32 \%, 88 \%-92 \%=31 \%, 93 \%-96 \%=23 \%$ and $97 \%-100 \%=28 \%$ (including inpatient deaths these figures were $43 \%, 37 \%, 32 \%$ and $40 \%$ ) (figure 3). The 93\%-96\% group had a significantly lower risk of death than the $88 \%-92 \%$ group, and overall (online supplemental table 5 includes further information relating to 1 -year mortality).

Table 2 Relationship between admission oxygen saturation group and inpatient mortality on univariate and multivariate analysis in patients receiving supplemental oxygen

\begin{tabular}{|c|c|c|c|c|c|c|c|}
\hline & \multirow[b]{2}{*}{ Died/total } & \multicolumn{3}{|c|}{ Univariate analysis } & \multicolumn{3}{|c|}{ Multivariate analysis } \\
\hline & & OR & $95 \% \mathrm{Cl}$ & $P$ value & OR & $95 \% \mathrm{Cl}$ & $P$ value \\
\hline \multicolumn{8}{|c|}{ Oxygen saturations } \\
\hline $88 \%-92 \%$ & $25 / 282$ & 1 & Reference & - & 1 & Reference & - \\
\hline $97 \%-100 \%$ & $38 / 223$ & 2.18 & 1.26 to 3.77 & 0.005 & 2.97 & 1.58 to 5.58 & 0.001 \\
\hline $93 \%-96 \%$ & $44 / 375$ & 1.40 & 0.83 to 2.37 & 0.205 & 1.98 & 1.09 to 3.60 & 0.025 \\
\hline $87 \%$ or less & $25 / 147$ & 2.17 & 1.19 to 3.96 & 0.011 & 1.36 & 0.69 to 2.71 & 0.377 \\
\hline NEWS2-sats & - & - & - & - & 1.22 & 1.12 to 1.34 & $<0.001$ \\
\hline DECAF & - & - & - & - & 2.45 & 2.03 to 2.95 & $<0.001$ \\
\hline
\end{tabular}

DECAF, dyspnoea, eosinopenia, chest X-ray consolidation, acidaemia, atrial fibrillation; NEWS2, National Early Warning Score. 
Table 3 Relationship between admission oxygen saturation group and inpatient mortality on univariate and multivariate analysis in patients receiving supplemental oxygen, with and without hypercapnia

\begin{tabular}{|c|c|c|c|c|c|c|c|}
\hline & \multirow[b]{2}{*}{ Died/ total } & \multicolumn{3}{|c|}{ Univariate analysis } & \multicolumn{3}{|c|}{ Multivariate analysis } \\
\hline & & OR & $95 \% \mathrm{Cl}$ & $P$ value & OR & $95 \% \mathrm{Cl}$ & P value \\
\hline Normocapnia & $\mathrm{N}=50 / 476$ & & & & & & \\
\hline \multicolumn{8}{|c|}{ Oxygen saturations } \\
\hline $88 \%-92 \%$ & $9 / 139$ & 1 & Reference & - & 1 & Reference & - \\
\hline $97 \%-100 \%$ & $16 / 105$ & 2.62 & 1.11 to 6.21 & 0.029 & 4.32 & 2.19 to 8.52 & $<0.001$ \\
\hline $93 \%-96 \%$ & $17 / 184$ & 1.47 & 0.63 to 3.41 & 0.373 & 2.31 & 1.17 to 4.58 & 0.016 \\
\hline $87 \%$ or less & $8 / 47$ & 2.93 & 1.06 to 8.14 & 0.039 & 2.08 & 1.01 to 4.25 & 0.046 \\
\hline NEWS2-sats & & - & - & - & 1.18 & 1.09 to 1.28 & $<0.001$ \\
\hline DECAF & & - & - & - & 2.74 & 2.36 to 3.17 & $<0.001$ \\
\hline Hypercapnia & $\mathrm{N}=81 / 551$ & & & & & & \\
\hline \multicolumn{8}{|c|}{ Oxygen saturations } \\
\hline $88 \%-92 \%$ & $15 / 143$ & 1 & Reference & - & 1 & Reference & - \\
\hline $97 \%-100 \%$ & $22 / 118$ & 1.90 & 0.93 to 3.90 & 0.079 & 2.28 & 0.99 to 5.21 & 0.052 \\
\hline $93 \%-96 \%$ & $27 / 191$ & 1.37 & 0.70 to 2.68 & 0.361 & 1.82 & 0.84 to 3.95 & 0.128 \\
\hline $87 \%$ or less & $17 / 99$ & 1.71 & 0.81 to 3.63 & 0.158 & 1.10 & 0.47 to 2.59 & 0.295 \\
\hline NEWS2-sats & & - & - & - & 1.26 & 1.12 to 1.43 & $<0.001$ \\
\hline DECAF & & - & - & - & 2.27 & 1.78 to 2.90 & $<0.001$ \\
\hline
\end{tabular}

DECAF, dyspnoea, eosinopenia, chest X-ray consolidation, acidaemia, atrial fibrillation; NEWS2, National Early Warning Score.

\section{DISCUSSION}

In 1027 patients admitted with ECOPD to 1 of 6 hospitals and treated with supplemental oxygen, in-hospital mortality was lowest in those with admission oxygen saturations between $88 \%$ and $92 \%$. This association was significant in patients with normocapnia in the primary and secondary analysis, but was unexpectedly not significant in the hypercapnic groups. This suggests that the practice of setting different target saturations based on $\mathrm{PaCO}_{2}$ is not justified. Mortality rates rose sequentially in the 93\%-96\% and 97\%-100\% groups. The higher mortality in patients with higher oxygen saturations cannot be explained by more severe acute illness and underlying frailty; this association remained after adjusting for baseline mortality risk assessed by DECAF and NEWS2 (excluding oxygen saturation). Furthermore, at 1 year, the $88 \%-92 \%$ cohort did not have better outcome to suggest a more favourable risk profile, though the 93\%-96\% groups had a significant lower risk of death. Both DECAF and NEWS2-sats scores were strong predictors of inpatient death in regression models, which confirms their prognostic value and suitability for risk adjustment. This, and the incremental relationship between oxygen saturations and mortality, supports a possible causal relationship between excess oxygen and mortality.
Guidelines from the BTS and endorsed by the ERS state that patients with ECOPD should initially have target saturations of $88 \%-92 \%$, though this recommendation is not consistently applied in clinical practice. ${ }^{12324}$ This may be partly due to two potentially incorrect assumptions: that oxygen levels which are modestly above the target range (93\%-96\%) are safe, and that patients with COPD with normal $\mathrm{PaCO}_{2}$ are immune to the harmful effects of oxygen.

International data on changes in oxygen practice are mostly lacking. UK national COPD audits show that while excess oxygen use in ECOPD remains a serious concern, ${ }^{1}$ practice is improving. The proportion of patients with hyperoxia $\left(\mathrm{PaO}_{2}\right.$ $>13 \mathrm{kPa} / 97.5 \mathrm{~mm} \mathrm{Hg}$ ) was $19 \%$ in 2003 and $16 \%$ in 2008 , falling to $8 \%$ in $2014 .{ }^{31}$ Of note, this fall followed the publication of the 2008 BTS oxygen guidelines which recommend target saturations of $88 \%-92 \%$ in most patients. In-hospital mortality fell from $7.8 \%$ in 2008 to $4.3 \%$ in 2014 , despite similar baseline characteristics and no substantial difference in other acute treatment (including NIV). Improved oxygen delivery may explain a substantial part of the fall in mortality. In our study, if a causal link is assumed, the number need to harm (NNH) to cause one death was 12 for the 97\%-100\% oxygen saturation group and 32 for the 93\%-96\% oxygen saturation

Table 4 Relationship between admission oxygen saturation group and inpatient mortality on univariate and multivariate analysis in patients without supplemental oxygen

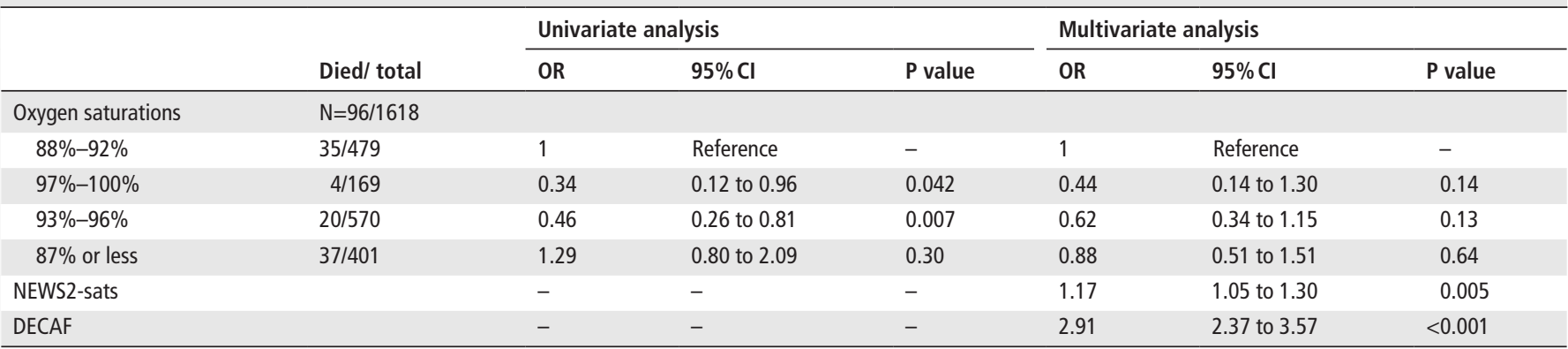

DECAF, dyspnoea, eosinopenia, chest X-ray consolidation, acidaemia, atrial fibrillation; NEWS2, National Early Warning Score. 


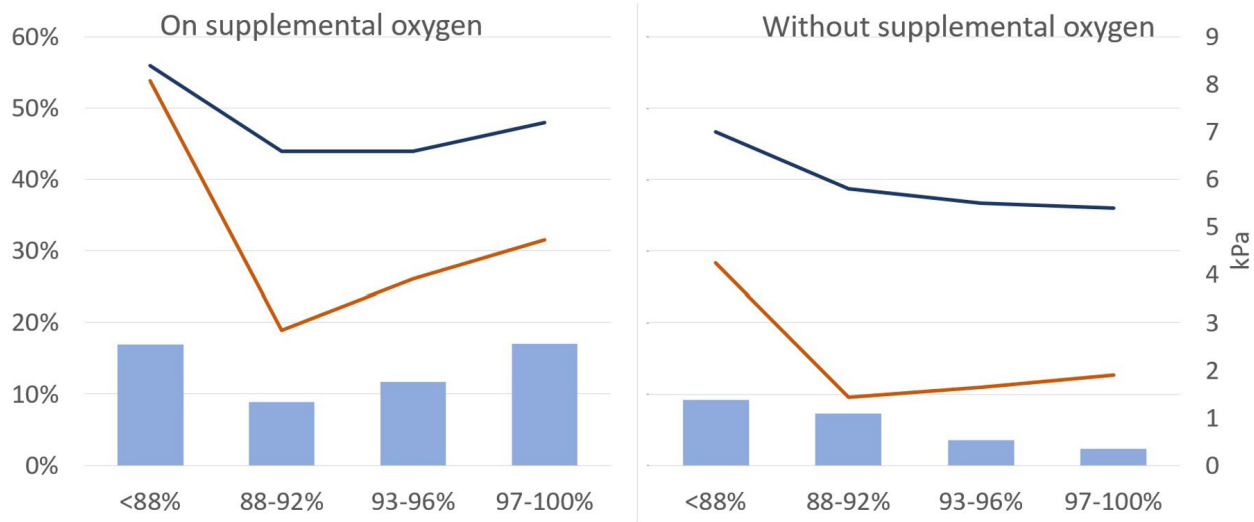

Died, $\%-\mathrm{pH}<7.35, \%-\mathrm{pCO} 2, \mathrm{kPa}$

Figure 2 Inpatient mortality, $\mathrm{pH}$ (per cent less than 7.35), and $\mathrm{pCO}_{2}(\mathrm{kPa})$ in those with (left) and without (right) supplemental oxygen. Those on supplemental oxygen (left) and those without (right) and the association between inpatient mortality, the proportion of patients with a pH of less than 7.35 and the $\mathrm{pCO}_{2}$ in $\mathrm{kPa}$. The per cent for mortality and those with acidaemia is shown on the left axis, and $\mathrm{kPa}$ is shown on the right.

group. The subgroup with normocapnia was at similar risk of harm $(\mathrm{NNH}=1$ in 11 and 1 in 36, respectively). Robinson et $a l^{18}$ have shown that oxygen therapy can lead to hypercapnia in patients with baseline normocapnia. We speculate that in our study the increased mortality risk in both the 97\%-100\% and 93\%-96\% normocapnic groups compared with the $88 \%-92 \%$ group may be due to inappropriate complacency in oxygen provision throughout admission.

Our results compliment those of the Australian cluster RCT: among patients with a clinical diagnosis of COPD, controlled oxygen therapy to target saturations 88\%-92\% from ambulance pick-up improved survival compared with high flow oxygen. ${ }^{20}$ In all patients, the magnitude of harm with high flow oxygen expressed as an OR was 2.52 which is similar to that seen in our group with oxygen saturations of 97\%-100\%. A recent systematic review showed that liberal oxygen use (defined as saturations 94\%-99\%) was associated with increased mortality in sepsis, critical illness, stroke, trauma, myocardial infarction and post cardiac arrest and an adverse dose response. ${ }^{21}$ Patients

\section{Normocapnic patients}

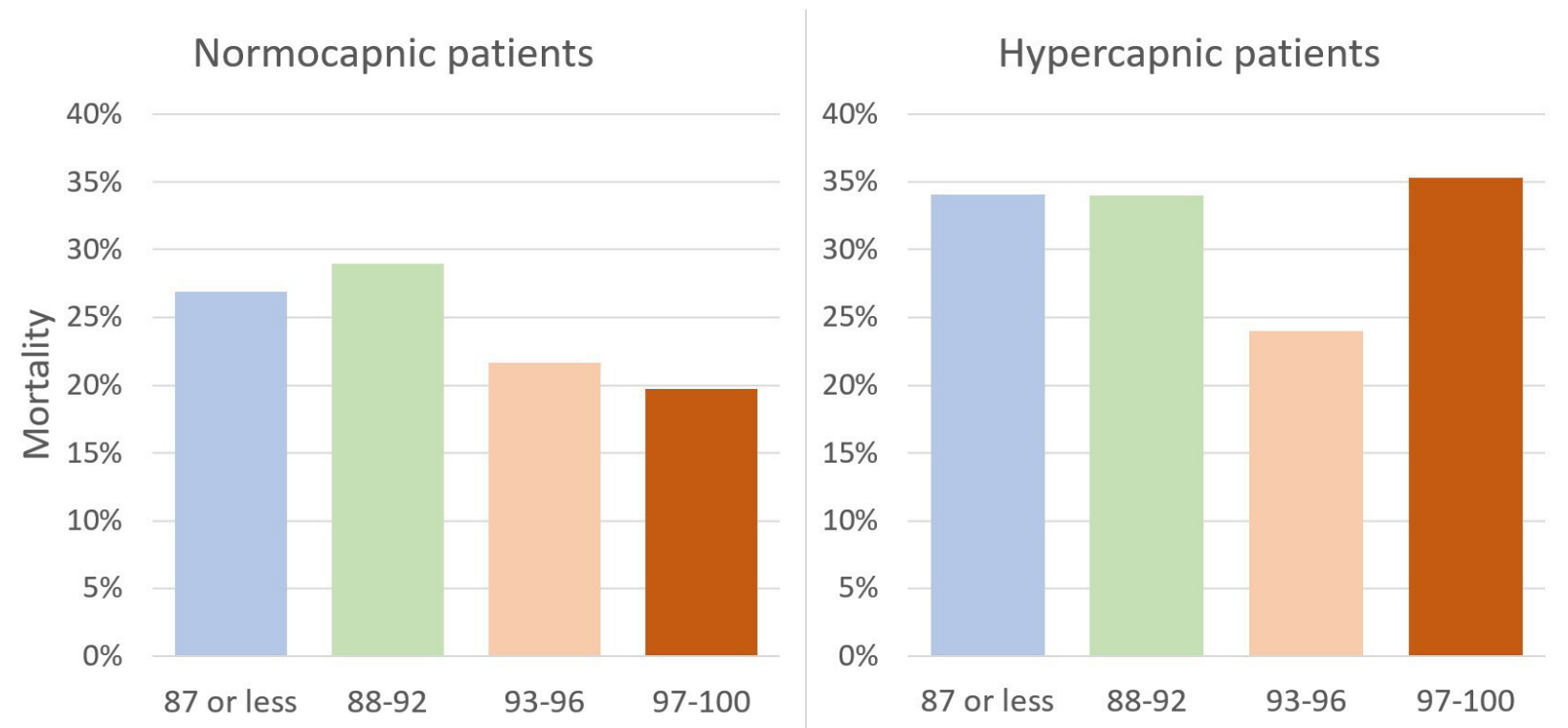

with ECOPD comprise one of the larger inpatient groups, often require oxygen and are frequently given oxygen above target saturations of $88 \%-92 \%$. Given the marked increased mortality rates with excess oxygen, this represents an important area globally for the prevention of death and morbidity.

Our study has several strengths. Our population were well described, with evidence of obstructive spirometry and a clinical history of COPD. Ethics approval was granted without the need for written consent as the study was observational, which allowed complete capture of consecutive patients. The need for written consent in observational studies may result in systematic bias, as the most unwell patients and most well patients are under-represented. More than $80 \%$ of patients were identified prospectively (some patients were identified within a discharge coding search that was performed on a wide range of respiratory admissions to ensure no patients were missed). Eligibility criteria were broad and rates of missing data were low, which supports the generalisability of our results. There was meticulous assessment of key prognostic indices allowing for accurate baseline

Figure 3 One-year mortality risk by admission oxygen saturation group in patients receiving supplemental oxygen, in patients that survived to discharge. 1-year mortality in patients with normocapnia $\left(\mathrm{PaCO}_{2} 45 \mathrm{~mm} \mathrm{Hg} / 6.0 \mathrm{kPa}\right.$ or less) and hypercapnia $\left(\mathrm{PaCO} \mathrm{O}_{2}>45 \mathrm{~mm} \mathrm{Hg} / 6 \mathrm{kPa}\right)$ by their admission oxygen saturation score, not including patients that died as an inpatient. 
risk adjustment. Data analyses, including methods to deal with missing data, were robust.

Our study has the following limitations. Indices were collected at admission only, and we did not have prehospital oxygen data for those admitted via ambulance. While audit data show that preadmission/admission is the main risk period for excess oxygen, longitudinal oxygen saturation data would have refined the analysis. A larger sample size would have provided clarity on the 93\%-96\% group, as the mortality difference seen with the $88 \%-92 \%$ overall group was significant in the primary analysis but not the secondary analysis. As with all observational studies, causation cannot be proven; while we used a robust regression model, the DECAF score includes $\mathrm{pH}$ which is associated with death as well as oxygen use and is therefore a potential cofounder. However, our findings are consistent with RCT data, and the validity of our findings is supported by the strength of the association, biological plausibility, a dose-dependent relationship and coherence with other clinical research. ${ }^{22}$ Given the harm seen with excess oxygen in patients with COPD, further RCTs in this area may be ethically unrealisable.

\section{CONCLUSION}

In conclusion, in patients with ECOPD receiving supplemental oxygen, those with oxygen saturations of $88 \%-92 \%$ had the highest survival compared with patients with higher oxygen saturations. Patients with normocapnia with oxygen saturations of 97\%-100\% and 93\%-96\% had worse outcome than the $88 \%-92 \%$ group. Randomised controlled studies have shown that the risk of excess oxygen includes patients with and without COPD, and likely included those without baseline hypercapnia. The extent of harm with excess oxygen is substantial, with numbers needed to cause death of 14 (subgroup with COPD), ${ }^{20} 20$ (patients with and without COPD) $)^{20}$ and 12.5 (patients with septic shock). ${ }^{32}$ In our study, we have shown that patients with normocapnia treated with excess oxygen have similar unacceptable levels of harm. We recommend a single rule for all patients with COPD, irrespective of the presence or absence of hypercapnia: oxygen saturations of $88 \%-92 \%$.

Widespread adoption of these target saturations from ambulance pick-up should simplify clinical pathways and reduce risk of excess oxygenation and death of patients with COPD.

Contributors The study was conceived by SCB and designed by CE, JS and SCB. CE and JW performed statistical analyses. All authors contributed to data analysis and interpretation, and all were involved in drafting the manuscript and approving the final version. The corresponding author attests that all listed authors meet authorship criteria and that no others meeting the criteria have been omitted.

Funding This research was funded by the UK Department of Health, Northumbria NHS Foundation Trust TRF programme, Breathe North appeal, and Novartis Pharmaceuticals UK (reference RES 12-CO44).

Competing interests All authors declare: IS has no conflicts of interest to declare. CE reports grants from National Institute of Health Research, outside of the submitted work. SB reports grants from National Institute of Health Research, Philips Respironics and from Pfizer Open Air, personal fees from Pfizer, AstraZeneca, and ResMed, and non-financial support from Boehringer Ingelheim and GlaxoSmithKline outside the submitted work. No author has financial relationships with any organisation that might have an interest in the submitted work.

Patient consent for publication Not required.

Ethics approval Ethics approval was granted by NRES Committee North East, UK.

Provenance and peer review Not commissioned; externally peer reviewed.

Data availability statement Data are available upon reasonable request and after approval of the research team.
Supplemental material This content has been supplied by the author(s). It has not been vetted by BMJ Publishing Group Limited (BMJ) and may not have been peer-reviewed. Any opinions or recommendations discussed are solely those of the author(s) and are not endorsed by BMJ. BMJ disclaims all liability and responsibility arising from any reliance placed on the content. Where the content includes any translated material, BMJ does not warrant the accuracy and reliability of the translations (including but not limited to local regulations, clinical guidelines, terminology, drug names and drug dosages), and is not responsible for any error and/or omissions arising from translation and adaptation or otherwise.

\section{ORCID iD}

Carlos Echevarria http://orcid.org/0000-0001-6916-8862

\section{REFERENCES}

1 The National Confidential Enquiry into Patient Outcome and Death. Inspiring Change. London, 2017. https://www.ncepod.org.uk/2017report2/downloads/ InspiringChange_FullReport.pdf

2 Alrawi YA, Potter JF, Myint PK. Uk national COPD resources and outcomes project (NCROP): 2008 national audit data presents an opportunity to highlight the areas for improvement in COPD care in the ageing population. COPD 2010;7:360-5.

3 Roberts CM, Stone RA, Buckingham RJ, et al. Acidosis, non-invasive ventilation and mortality in hospitalised COPD exacerbations. Thorax 2011;66:43-8.

4 Kallstrom TJ, American Association for Respiratory Care (AARC). AARC Clinical Practice Guideline: oxygen therapy for adults in the acute care facility--2002 revision \& update. Respir Care 2002;47:717-20.

5 Casaubon LK, Boulanger J-M, Blacquiere D, et al.

Canadian Stroke Best Practice Recommendations: Hyperacute Stroke Care Guidelines, Update 2015. Int J Stroke 2015;10:924-40.

6 Nikolaou NI, Welsford M, Beygui F, et al. Part 5: acute coronary syndromes: 2015 international consensus on cardiopulmonary resuscitation and emergency cardiovascular care science with treatment recommendations. Resuscitation 2015;95:e121-46.

7 Helmerhorst HJF, Roos-Blom M-J, van Westerloo DJ, et al. Association between arterial hyperoxia and outcome in subsets of critical illness: a systematic review, metaanalysis, and meta-regression of cohort studies. Crit Care Med 2015;43:1508-19.

8 Moga C, Chojecki D. Oxygen therapy in acute care settings. Institute of Health Economics 2016.

9 Siemieniuk RAC, Chu DK, Kim LH-Y, et al. Oxygen therapy for acutely ill medical patients: a clinical practice guideline. BMJ 2018;363:k4169.

10 O'Driscoll BR, Howard LS, Earis J, et al. BTS guideline for oxygen use in adults in healthcare and emergency settings. Thorax 2017;72:ii1-90.

11 Royal College of Physicians. National early warning score (NEWS) 2: standardising the assessment of acute-illness severity in the NHS. London; 2017.

12 Kane B, Decalmer S, Ronan O'Driscoll B. Emergency oxygen therapy: from guideline to implementation. Breathe 2013;9:246-53.

13 Wedzicha JA, Miravitlles M, Hurst JR, et al. Management of COPD exacerbations: a European Respiratory Society/American Thoracic Society guideline. Eur Respir J 2017;49:1600791.

14 GOLD. Global initiative for chronic obstructive lung disease; 2019

15 Beasley R, Chien J, Douglas J, et al. Thoracic Society of Australia and New Zealand oxygen guidelines for acute oxygen use in adults: 'Swimming between the flags'. Respirology 2015;20:1182-91

16 Brill SE, Wedzicha JA. Oxygen therapy in acute exacerbations of chronic obstructive pulmonary disease. Int J Chron Obstruct Pulmon Dis 2014;9:1241-52.

17 Robinson TD, Freiberg DB, Regnis JA, et al. The role of hypoventilation and ventilationperfusion redistribution in oxygen-induced hypercapnia during acute exacerbations of chronic obstructive pulmonary disease. Am J Respir Crit Care Med 2000;161:1524-9.

18 Hafner S, Beloncle F, Koch A, et al. Hyperoxia in intensive care, emergency, and peri-operative medicine: Dr. Jekyll or Mr. Hyde? A 2015 update. Ann Intensive Care 2015;5:42.

19 Stub D, Smith K, Bernard S, et al. Air versus oxygen in ST-segment-elevation myocardial infarction. Circulation 2015;131:2143-50.

20 Austin MA, Wills KE, Blizzard L, et al. Effect of high flow oxygen on mortality in chronic obstructive pulmonary disease patients in prehospital setting: randomised controlled trial. BMJ 2010;341:c5462.

21 Chu DK, Kim LH-Y, Young PJ, et al. Mortality and morbidity in acutely ill adults treated with liberal versus conservative oxygen therapy (IOTA): a systematic review and metaanalysis. Lancet 2018;391:1693-705

22 European Respiratory Society. An international comparison of COPD care in Europe, results of the first European COPD audit; 2012

23 O'Driscoll BR, Rudenski A, Turkington PM, et al. An audit of hypoxaemia, hyperoxaemia, hypercapnia and acidosis in blood gas specimens. Eur Respir $J$ 2012;39:219-21.

24 Kelly CA, Maden M. How do health-care professionals perceive oxygen therapy? A critical interpretive synthesis of the literature. Chron Respir Dis 2015;12:11-23.

25 Steer J, Gibson J, Bourke SC. The DECAF score: predicting hospital mortality in exacerbations of chronic obstructive pulmonary disease. Thorax 2012;67:970-6. 
26 Echevarria C, Steer J, Heslop-Marshall K, et al. Validation of the DECAF score to predict hospital mortality in acute exacerbations of COPD. Thorax 2016;71:133-40.

27 Echevarria C, Steer J, Heslop-Marshall K, et al. The PEARL score predicts 90-day readmission or death after hospitalisation for acute exacerbation of COPD. Thorax 2017;72:686-93.

28 Rubin DB. Multiple imputation after 18+ years. J Am Stat Assoc 1996;91:473-89.

29 Huang A. Mean-parametrized Conway-Maxwell-Poisson regression models for dispersed counts. Stat Modelling 2017;17:359-80.
30 VanderWeele TJ, Ding P. Sensitivity analysis in observational research: introducing the E-Value. Ann Intern Med 2017;167:268-74.

31 Stone RA, Holzhauer-Barrie J, Lowe D. COPD: who cares matters. National chronic obstructive pulmonary disease (COPD) audit programme: clinical audit of COPD exacerbations admitted to acute units in England and Wales 20142015.

32 Asfar P, Schortgen F, Boisramé-Helms J, et al. Hyperoxia and hypertonic saline in patients with septic shock (HYPERS2S): a two-by-two factorial, multicentre, randomised, clinical trial. Lancet Respir Med 2017;5:180-90. 